\title{
Recapitulation of spinal motor neuron-specific disease phenotypes in a human cell model of spinal muscular atrophy
}

\author{
Zhi-Bo Wang ${ }^{1}$, Xiaoqing Zhang ${ }^{2}$, Xue-Jun Li ${ }^{1,3}$ \\ ${ }^{I}$ Department of Neuroscience, the University of Connecticut Health Center, 263 Farmington Avenue, Farmington, CT 06030-3401, \\ USA; ${ }^{2}$ Department of Regenerative Medicine, Tongji University School of Medicine, Shanghai 200092, China, ${ }^{3}$ Stem Cell Institute, \\ the University of Connecticut Health Center, Farmington, CT 06030, USA
}

Establishing human cell models of spinal muscular atrophy (SMA) to mimic motor neuron-specific phenotypes holds the key to understanding the pathogenesis of this devastating disease. Here, we developed a closely representative cell model of SMA by knocking down the disease-determining gene, survival motor neuron (SMN), in human embryonic stem cells (hESCs). Our study with this cell model demonstrated that knocking down of SMN does not interfere with neural induction or the initial specification of spinal motor neurons. Notably, the axonal outgrowth of spinal motor neurons was significantly impaired and these disease-mimicking neurons subsequently degenerated. Furthermore, these disease phenotypes were caused by SMN-full length (SMN-FL) but not SMN- $\Delta 7$ (lacking exon 7) knockdown, and were specific to spinal motor neurons. Restoring the expression of SMN-FL completely ameliorated all of the disease phenotypes, including specific axonal defects and motor neuron loss. Finally, knockdown of SMNFL led to excessive mitochondrial oxidative stress in human motor neuron progenitors. The involvement of oxidative stress in the degeneration of spinal motor neurons in the SMA cell model was further confirmed by the administration of $\mathrm{N}$-acetylcysteine, a potent antioxidant, which prevented disease-related apoptosis and subsequent motor neuron death. Thus, we report here the successful establishment of an hESC-based SMA model, which exhibits disease gene isoform specificity, cell type specificity, and phenotype reversibility. Our model provides a unique paradigm for studying how motor neurons specifically degenerate and highlights the potential importance of antioxidants for the treatment of SMA.

Keywords: spinal muscular atrophy; human embryonic stem cells; spinal motor neuron; RNA interference; disease modeling Cell Research (2013) 23:378-393. doi:10.1038/cr.2012.166; published online 4 December 2012

\section{Introduction}

Spinal muscular atrophy (SMA) is the leading genetic cause of death in infants and toddlers, characterized by the selective loss of spinal motor neurons and subsequent muscle atrophy [1]. The SMA-determining gene, survival motor neuron $(S M N)$, was identified in 1995 [2]. Different from rodents that have only one $S M N$ gene, humans have two $S M N$ genes: a telomeric copy (SMN1)

Correspondence: Xue-Jun Li

Tel: 1+860-679-3026; Fax: 1+860-679-8766

E-mail: xjli@uchc.edu

Received 15 April 2012; revised 24 September 2012; accepted 1 November 2012; published online 4 December 2012 and an inverted centromeric copy (SMN2) [3]. The SMN1 gene generates full-length transcripts (SMN-FL) and functional SMN protein. Although the sequence of the $S M N 2$ gene is basically identical to that of SMN1 except for 5 base pair changes, the $S M N 2$ gene has disrupted splicing of exon 7, which results in the generation of transcripts $(90 \%)$ lacking exon $7(\mathrm{SMN}-\Delta 7)[4,5]$. SMN$\Delta 7$ protein is unstable and degrades quickly. More than $95 \%$ of SMA patients have mutations in the SMN1 gene, yet have normal or even extra copies of the SMN2 gene [6]. Since the SMN2 gene produces only a low level of full-length functional SMN protein, SMN2 cannot fully compensate for the homozygous loss of SMN1 in SMA patients. Therefore, SMA patients experience a loss of proper SMN function, which leads to subsequent motor 
neuron loss and clinical symptoms.

How a decreased level of SMN protein, a ubiquitously expressed protein, results in specific degeneration of spinal motor neurons has remained a mystery. SMN protein is concentrated in prominent dot-like structures (or gems) in the nucleus of many cell types $[7,8]$. Nuclear SMN is critical in the assembly of many different classes of small ribonucleoprotein particles (snRNPs); thus, SMN has a housekeeping role in snRNP biogenesis and in pre-mRNA splicing $[9,10]$. Although a decrease of snRNP biogenesis has been observed in vitro [11], its connection to pathological changes in spinal motor neurons in SMA is not understood. Recent studies reveal that, besides being located in the nucleus, SMN protein also accumulates in the dendrites and axons of neurons and is associated with microtubules $[12,13]$. SMN protein can regulate $\beta$-actin, a major component of outgrowing axons [14]. In primary neural culture from SMA animals, spinal motor neurons exhibited reduced growth cone size, reduced $\beta$-actin levels, and shorter axons [14]. Although studies suggest an emerging role of SMN in the axonal and synaptic functions of neurons, it remains unclear whether these functions underlie the specific degeneration of motor neurons and how these functions are regulated. Considering that humans are special in having two SMN genes that exhibit unique alternative splicing, a human cell model of SMA will be invaluable in exploring these questions and investigating the role of different SMN transcripts for the function of human motor neurons.

Human embryonic stem cells (hESCs), isolated from the inner cell mass of a preimplantation blastocyst, can produce all somatic cell types [15]. Based on developmental principles, hESCs have been differentiated into neural lineage and different neuronal subtypes, including spinal motor neurons [16-18] and midbrain dopaminergic neurons [19-21]. Spinal motor neurons, which are hard to culture and maintain from primary tissues, can be generated in large quantity from ESCs. Spinal motor neurons have been generated from induced pluripotent stem cells (iPSCs) by using a similar strategy [22-24], but variations in neural induction and/or motor neuron generation between different iPSC lines have been reported. Through the generation of SMA patient-specific iPSCs, recent studies revealed that the proportion of motor neurons derived from SMA iPSCs significantly decreased [25, 26]; however, whether this reduction results from insufficient spinal motor neuron specification or neuronal degeneration after specification remains elusive. Moreover, demonstration of disease-specific pathogenesis and cell type-specific phenotypes in human SMA models awaits further investigation. In the present study, to exclude the cell line variation between different iPSC lines, we es- tablished a cell-based SMA in vitro model by knocking down the SMN gene in hESCs. Our hESC-based SMA model closely recapitulates disease-specific phenotypes; more importantly, these disease phenotypes are cell type specific and reversible with the reintroduction of the $S M N-F L$ gene. Furthermore, taking advantage of this culture model, we discovered that mitochondrial oxidative stress is implicated in the functional defects in our SMA model and that antioxidants represent a potential strategy to prevent spinal motor neuron degeneration in SMA.

\section{Results}

Expression of SMN isoforms during motor neuron differentiation from $\mathrm{hESCS}$

Humans are unique in that they have two $S M N$ genes and generate two different transcripts, SMN-FL and SMN- $\Delta 7$. To better understand the function of $S M N$ in human motor neuron development, we first examined the temporal expression of SMN-FL and SMN- $\Delta 7$ transcripts at different time points during the differentiation of motor neurons from hESCs. Spinal motor neurons were generated using an established system as we described previously $[17,27]$. Briefly, hESCs were induced to neural lineage by generating ESC aggregations (so called embryoid body) and then culturing them in neural medium. Early neuroepthelial (NE) cells were formed around 8-10 days after differentiation from hESCs, when they exhibited columnar morphological changes and started to organize into rosette-like structures (Figure 1A). To efficiently generate caudalized neural precursors, we added retinoic acid (RA) to the early (primitive) NE cells. After 1 week of treatment, the caudalized NE cells were isolated, suspended as neurospheres, and treated with RA and purmorphamine (an agonist for the sonic hedgehog pathway) to generate spinal motor neurons. As we reported earlier [17, 27], OLIG2 ${ }^{+}$spinal motor neuron progenitors and $\mathrm{HB}^{+}$postmitotic neurons were efficiently generated at about 4 and 5 weeks after differentiation, respectively (Figure 1A). These motor neurons then matured in vitro as indicated by the expression of mature motor neuron markers and the formation of synaptic connections with other cells $[17,27]$. Using this paradigm, we then collected samples at representative stages during motor neuron differentiation and analyzed the expression of SMN-FL and SMN- $\Delta 7$. As shown by reverse transcriptase quantitative PCR (RT-qPCR), at different time points during neural and motor neuron differentiation, the expression of SMN-FL and SMN- $\Delta 7$ was significantly increased (Figure 1B and 1C). Since the condition in generating spinal motor neurons induced both spinal motor neurons and spinal interneurons [27], we further com- 
pared the expression of SMN in spinal motor neuronenriched cultures (RA plus purmorphamine) with that in spinal interneuron-enriched cultures [27] (RA alone) at the peak of SMN expression (day 26). The expression of Olig2, a marker for motor neuron progenitors, was much higher in motor neuron-enriched cultures than that in interneuron cultures (Supplementary information, Figure S1), confirming the identities of these cultures. Interestingly, the expression of SMN-FL mRNA was also much higher in motor neuron-enriched culture, suggesting that the increase in SMN-FL expression may originate, at least partially, from its higher expression in spinal motor neurons.

RA efficiently caudalized the neural cells and in the absence of RA and other caudalizing factors, rostral neural cells (forebrain neurons) were generated in basic medium $[28,29]$. To test whether the increase of SMN specifically occurs in caudalized neural cells during differentiation, we next examined the expression of SMN during the differentiation of forebrain neurons in basic conditions. At the same time points as those for spinal motor neuron differentiation, samples were collected and the expression of SMN-FL and SMN- $\Delta 7$ was examined (basic condition in Figure 1B and 1C). Interestingly, the expression of SMN did not significantly change during differentiation of forebrain neurons and was maintained at a similar level as that in hESCs (Figure 1B and 1C). The same batch of hESCs was used to generate either forebrain neurons or spinal motor neurons; thus the basic level of SMN on day 0 and day 10 were the same, facilitating the comparison. Different batch of hESCs were used to repeat the experiment and similar results were observed. Together, these data suggest that the expression of SMN increased significantly during the differentiation of hESCs into spinal motor neurons, but not forebrain neurons.

Establishing stable hESC lines with the knockdown of $S M N-F L$ and $S M N-\triangle 7$

SMA is caused by the decreased level of functional SMN protein. The increased expression of $S M N$, a housekeeping gene, in spinal motor neuron progenitors

A
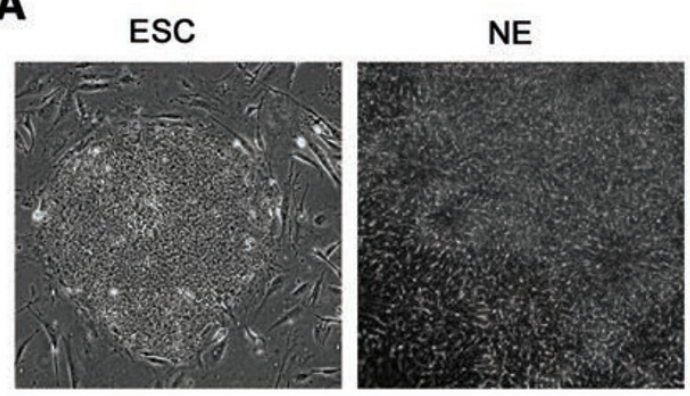

MN progenitor

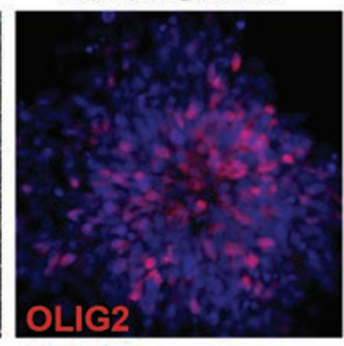

MN

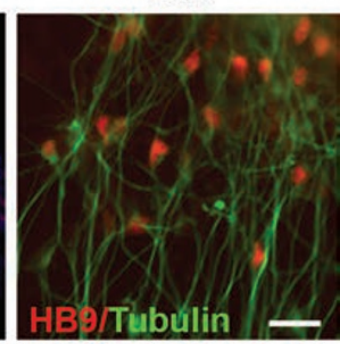

B

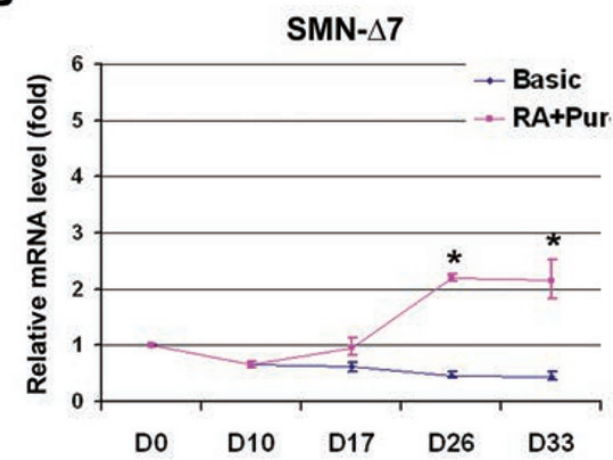

C

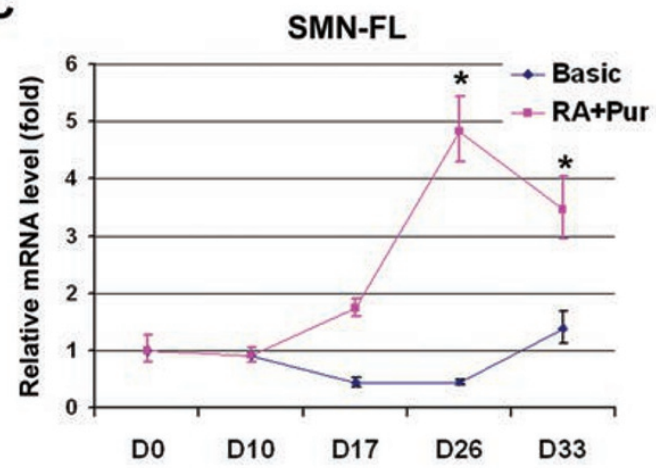

Figure 1 Expression of SMN-FL and $S M N-\Delta 7$ transcripts during motor neuron differentiation from hESCs. (A) Images showing important stages during spinal motor neuron differentiation from hESCs including neuroepithelial (NE) cells (10 days, D10), OLIG2-positive motor neuron (MN) progenitors (26 days, D26), and HB9-positive motor neurons (33 days, D33). Blue indicates Hoechst-stained nuclei. Scale bar, $20 \mu \mathrm{m}$. (B, C) Expression of SMN- $\Delta 7$ and SMN-FL transcripts at different times during differentiation of spinal motor neurons (RA + purmorphamine treatment, i.e., RA + Pur group) or forebrain neurons (basic conditions) from hESCs was examined by RT-qPCR. Data are presented as Mean \pm SD. ${ }^{*} P<0.05$ versus basic groups. 
and motor neurons indicates that SMN may exert some cell type-specific roles in spinal motor neuron development. To further elucidate the role of SMN, especially two different SMN transcripts, in the functions of motor neurons, we established hESC lines with the knockdown of SMN-FL or SMN- $\Delta 7$ (Figure 2A). This was achieved through a combination of lentiviral infection and RNA interference (RNAi) (Supplementary information, Figure S2). An shRNA targeting luciferase, which is not expressed in mammalian cells, was used as a control. Expression of green fluorescent protein (GFP) and blasticidin (BSD), a drug selection marker, facilitated the selection of pure transduced clones. After selection, uniform $\mathrm{GFP}^{+}$hESC clones containing shRNAs for luciferase, SMN-FL, and SMN- $\Delta 7$ were established (Figure $2 \mathrm{~A}$ ).

Next, we tested whether the expression of SMN can be efficiently knocked down and more importantly, whether the knockdown effect can be maintained when these ESCs differentiate into spinal motor neurons. Both SMN-FL RNAi and SMN- $\Delta 7$ RNAi hESC lines, as well as the luciferase RNAi hESC line (control), were differentiated into spinal motor neurons using the same paradigm as described $[17,27]$. In long-term culture, motor neurons expressed choline acetyltransferase (ChAT), an enzyme for synthesizing the neurotransmitter (Figure 2B). The $\mathrm{ChAT}^{+}$motor neurons were also doublestained with Bassoon, a presynaptic marker, or vesicular glutamate transporter 1 (vGLUT1), a protein expressed by excitatory synaptic terminals (Figure 2B-2D). This suggests that the spinal motor neurons matured and made synaptic connections with other cells in the culture. At different stages during differentiation, the expression of shRNA, as indicated by GFP, was not silenced (Figure $2 \mathrm{~A}$ and $2 \mathrm{~B}$ ). The mRNA expression of SMN-FL was significantly lower in SMN-FL RNAi hESCs, but not in SMN- $\triangle 7$ RNAi hESCs, than that in control cells (Figure $2 \mathrm{E})$. Conversely, the mRNA expression of SMN- $\Delta 7$ was specifically decreased in SMN- $\Delta 7$ RNAi hESCs (Figure $2 \mathrm{E}$ ), suggesting that hESCs with the specific knockdown of two SMN transcripts were established. The knockdown effect of SMN-FL (Figure 2G) and SMN$\Delta 7$ mRNAs (Supplementary information, Figure S3) was also maintained during motor neuron differentiation. At the protein level, the expression of SMN-FL protein in SMN-FL RNAi hESCs (Figure 2F) and in subsequent differentiated motor neurons (Figure $2 \mathrm{H}$ ) was significantly decreased compared to that in either the SMN- $\Delta 7$ RNAi or the luciferase RNAi cells. These data suggest that the establishment of hESC lines with the knockdown of specific SMN transcripts was successful, and that the knockdown effect can be maintained during differentiation into spinal motor neurons.
Knockdown of SMN does not alter neural induction and motor neuron specification

Neural induction is the first step toward the generation of spinal motor neurons. We next examined whether knockdown of $S M N$ had any effect on neural induction. In our culture, neural lineage was induced as indicated by the appearance of columnar NE cells about 8-10 days after differentiation. Ten days after differentiation from both SMN-FL RNAi and SMN- $\Delta 7$ RNAi hESCs, columnar cells appeared and formed rosette-like structures, similar to control cells (Figure 3A). To further compare the efficiency in generating NE cells, we examined the proportion of $\mathrm{PAX}^{+}$cells on day 10 after differentiation by fluorescent activated cell sorting (FACS) analysis. The generation of $\mathrm{PAX}^{+} \mathrm{NE}$ cells was highly efficient (over 90\%) in all three groups (control, SMN-FL RNAi, and SMN- $\triangle 7 \mathrm{RNAi}$ (Figure $3 \mathrm{~A}$ ). These findings suggest that knockdown of SMN does not alter neural induction and that NE cells can be efficiently generated from both SMN-FL- and SMN- $\Delta 7$-knockdown hESCs.

To test whether knockdown of SMN affects the specification of spinal motor neurons, NE cells were then induced to spinal motor neurons by the application of RA and purmorphamine as described earlier [17, 27]. $\mathrm{OLIG}^{+}$spinal motor neuron progenitors and $\mathrm{HB}^{+}$postmitotic neurons were generated at about 26 and 33 days after differentiation from SMN-knockdown hESCs, respectively, similar to the temporal differentiation in control and normal hESCs (Figure 3B). Further comparison of the proportion of OLIG2- and HB9-positive cells on day 26 and day 33 after differentiation showed that there were no significant differences between SMN-FL knockdown, SMN- $\Delta 7$ knockdown, and control groups (Figure 3C). These data suggest that knockdown of SMN-FL or SMN- $\Delta 7$ affects neither the temporal differentiation of spinal motor neurons nor the initial generation of spinal motor neurons from hESCs.

Knockdown of SMN-FL leads to impaired axonal outgrowth specifically in spinal motor neurons

Spinal motor neurons are large projection neurons with long axons. We next examined whether knockdown of SMN affects axonal outgrowth of human motor neurons and whether this happens in the early stage during motor neuron differentiation. Motor neuron-enriched clusters (1 month after differentiation) were dissociated and plated on polyornithine/laminin-coated coverslips. Two days after plating, these coverslips were fixed and subjected to Tau immunostaining to analyze axonal outgrowth (Figure 4A and 4B). In control group (luciferase RNAi), long $\mathrm{TAU}^{+}$processes grew out from the neuron soma cluster (Figure 4A) and were double-stained with 
A

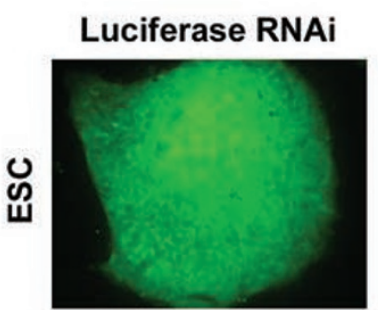

SMN-FL RNAi

SMN- $\triangle 7$ RNAi
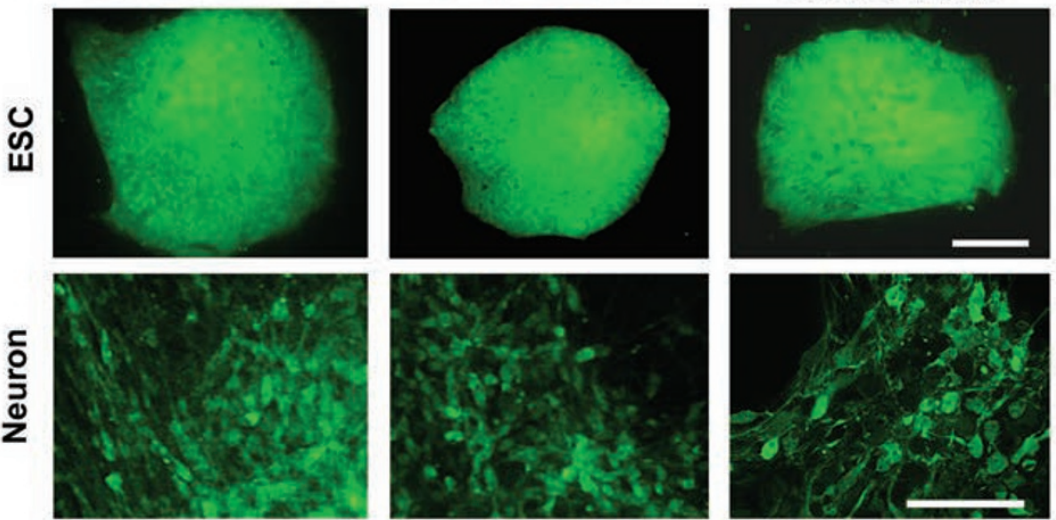

B

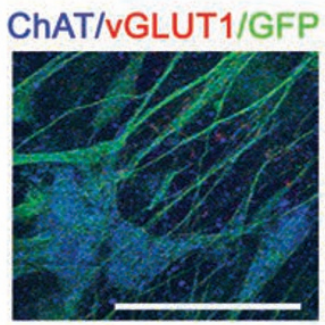

C

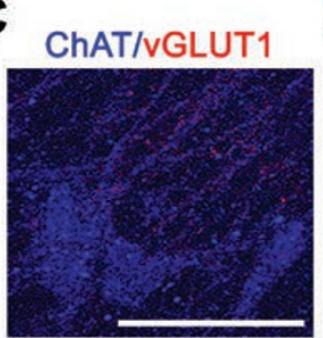

D
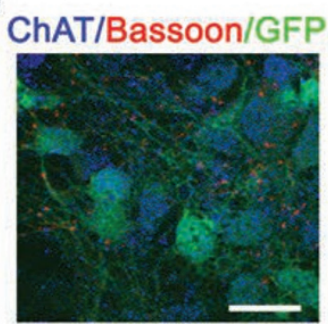

E

SMN expression in ESCs

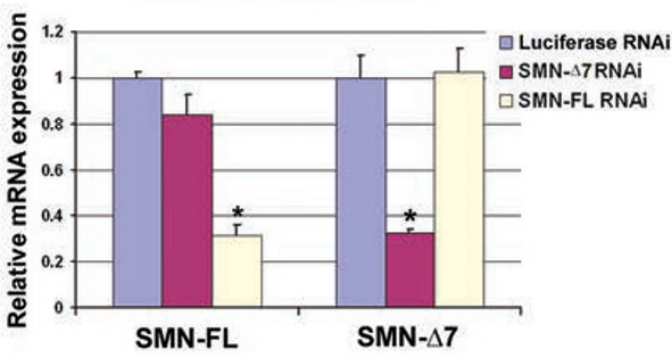

G

SMN-FL Knockdown Efficiency
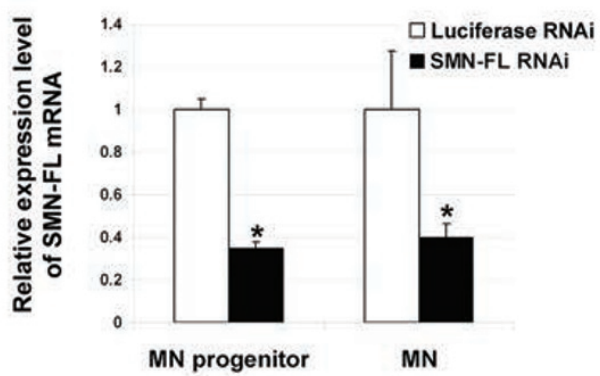

$\mathbf{F}$

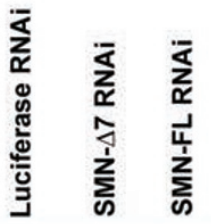

SMN-FL

Actin

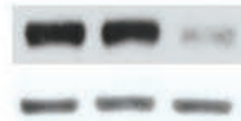

ESC Stage

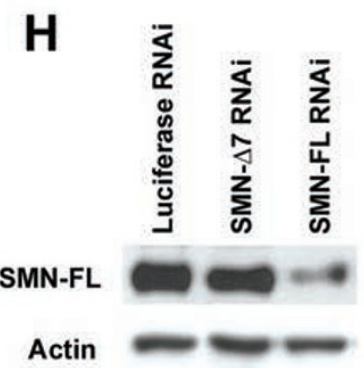

MN Stage

Figure 2 Generation of hESC lines with the stable knockdown of SMN-FL and SMN- $\Delta 7$. (A) The expression of shRNA, as indicated by GFP, was maintained during motor neuron differentiation from SMN-FL RNAi, SMN- $\triangle 7$ RNAi, and luciferase RNAi hESCs. (B) Eight weeks after differentiation from luciferase RNAi hESCs, motor neurons matured in the culture and expressed ChAT. The ChAT ${ }^{+}$cells formed presynaptic clusters as indicated by the double staining of ChAT with vGLUT1 (B, C) or Bassoon (D). (E) qPCR and (F) western blot showing the differential knockdown of SMN-FL or SMN- $\Delta 7$ in the hESC lines containing SMN-FL RNAi or SMN- $\triangle 7$ RNAi. The expression of SMN-FL mRNA (G) and protein (H) in motor neuron progenitors and postmitotic neurons derived from SMN-FL RNAi hESCs decreased significantly compared with that in the control group. Data are presented as mean \pm SD. ${ }^{*} P<0.05$ versus control (luciferase RNAi) group. Scale bars, $50 \mu \mathrm{m}$ (A-C) and $20 \mu \mathrm{m}$ (D). 
A
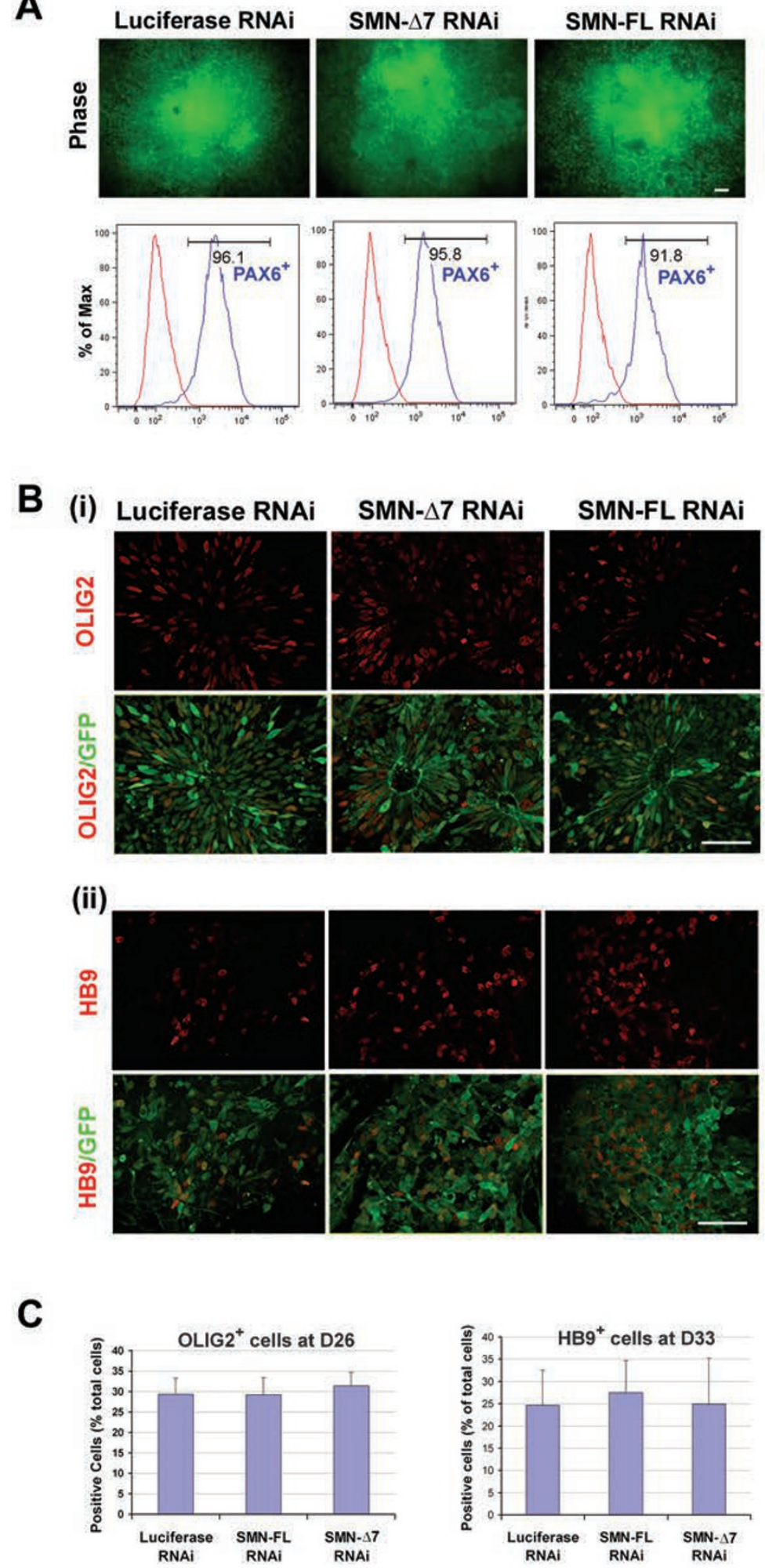

Figure 3 Effect of SMN-FL and SMN- $\Delta 7$ knockdown on neural induction and motor neuron specification. (A) The generation of columnar NE cells and the proportion of $\mathrm{PAX}^{+}$cells were similar among NE cells derived from SMN-FL RNAi, SMN- $\Delta 7$ RNAi, and luciferase RNAi hESCs. (B) Immunostaining showing the generation of OLIG2 ${ }^{+}$motor neurons progenitors (i) and $\mathrm{HB9}^{+}$postmitotic motor neurons (ii) from SMN-FL RNAi, SMN- 7 RNAi, and luciferase RNAi hESCs. (C) Neither the knockdown of SMN-FL nor SMN- $\Delta 7$ affects the specification of spinal motor neurons, as similar proportions of OLIG2 ${ }^{+}$progenitors or $\mathrm{HB}^{+}$motor neurons were generated in these cultures. Data are presented as mean $\pm \mathrm{SD}$. Scale bar, $50 \mu \mathrm{m}$. 
A
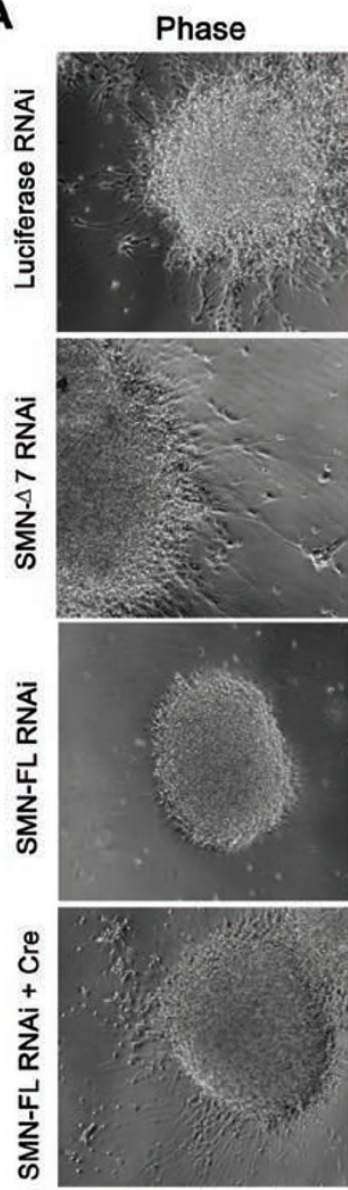

F

¿
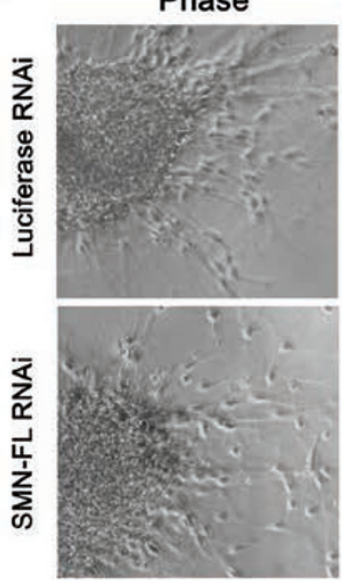

HB9/TAU
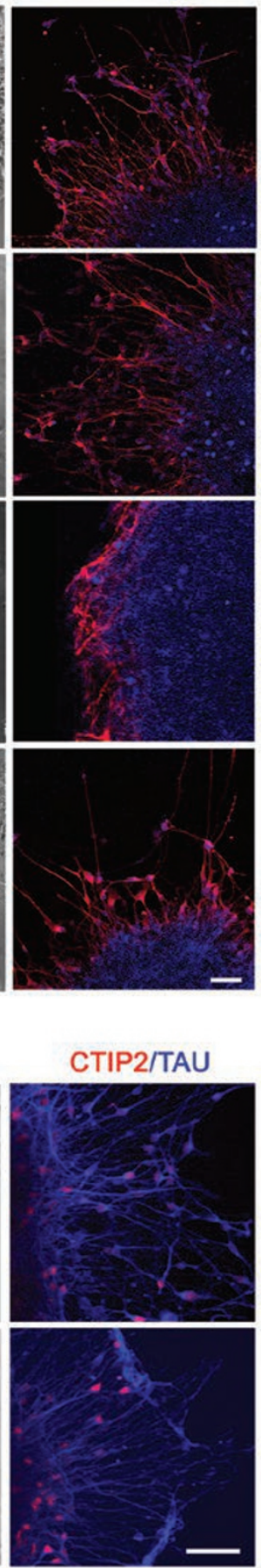

B

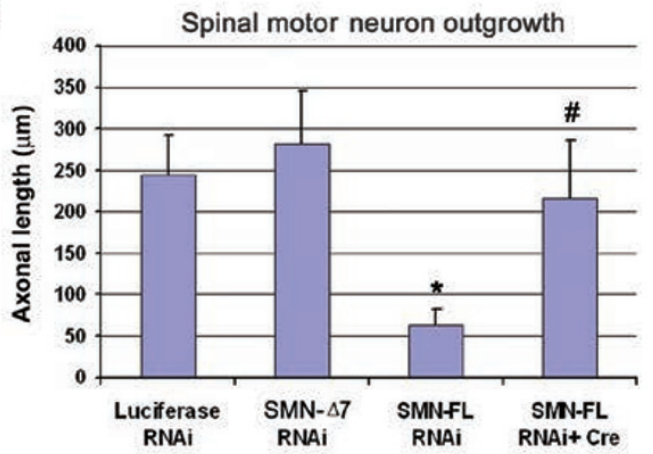

C

TAU/Bassoon/GFP

D TAU/Bassoon
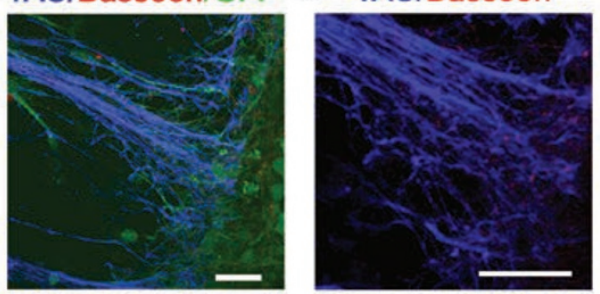

E

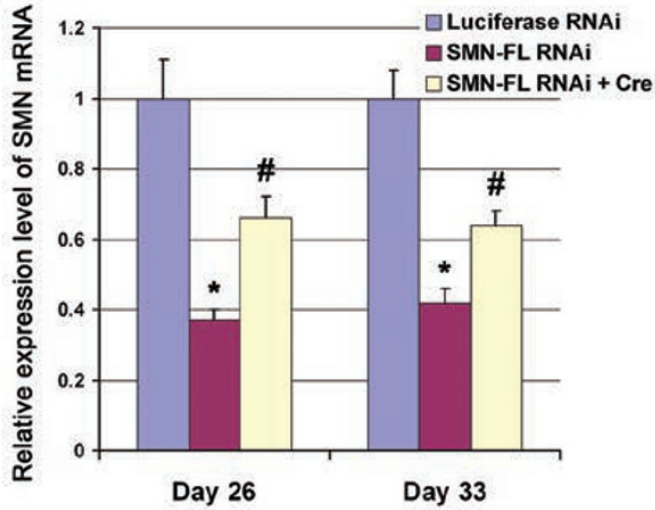

G

CTIP2 $^{+}$forebrain neuron outgrowth

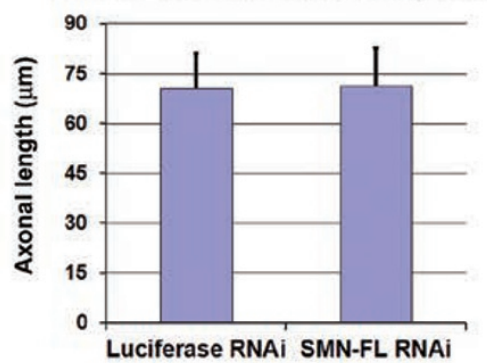

Figure 4 Human motor neurons derived from SMN-FL RNAi hESCs exhibited specific axonal outgrowth defects. (A) Axonal outgrowth, as indicated by the length of $\mathrm{TAU}^{+}$axons, was compared among spinal motor neurons ( $\mathrm{HB9}{ }^{+}$) derived from $\mathrm{SMN}^{-}$ FL RNAi, SMN- 7 RNAi, and luciferase RNAi hESCs. (B) Knockdown of SMN-FL resulted in a specific decrease in axonal length, which was rescued by the excision of shRNA through the application of Cre. (C, D) The TAU ${ }^{+}$axons growing out of a cluster in motor neuron cultures also expressed Bassoon, a presynaptic marker. $\mathbf{D}$ is the magnification of the center area in $\mathbf{C}$. (E) The application of Cre to NE cells (on day 16) restored the expression of SMN in motor neuron cultures. Meanwhile, the axonal outgrowth defects were also ameliorated in these Cre-treated cultures. (F) CTIP2 ${ }^{+}$forebrain neurons were generated from luciferase RNAi and SMN-FL RNAi hESCs and their axonal outgrowth was compared. (G) The axonal length of CTIP2 ${ }^{+}$ neurons was similar in SMN-knockdown and control groups. Data are presented as mean $\pm \mathrm{SD}$. ${ }^{*} P<0.05$ versus control (luciferase RNAi) group, ${ }^{\#} P<0.05$ versus SMN-FL RNAi group. Scale bars, $50 \mu \mathrm{m}$ (A, F) and $20 \mu \mathrm{m}$ (C, D). 
Bassoon (Figure 4C and 4D), revealing that they are axons. Notably, axons projected from spinal motor neuronenriched cultures (RA + purmorphamine treatment) were much longer than that from spinal interneuron cultures (RA alone group, Supplementary information, Figure S4), suggesting the cell type specificity of these long axons. We then compared the axonal outgrowth between control and SMN-knockdown groups. Although SMNFL knockdown did not influence the early specification of $\mathrm{HB}^{+}$postmitotic motor neurons, we consistently observed reduced axonal outgrowth in spinal motor neurons derived from SMN-FL RNAi hESCs compared with that from luciferase RNAi hESC (Figure 4A and 4B). In contrast, knockdown of SMN- $\Delta 7$ did not alter the axonal length of $\mathrm{HB}^{+}$spinal motor neurons (Figure $4 \mathrm{~A}$ and 4B), suggesting that the phenotype is SMN-FL isoform specific. In addition, knockdown of SMN-FL in the other hESC line, H1, led to a similar axonal outgrowth defect in spinal motor neuron cultures on day 33 after differentiation (Supplementary information, Figure S5), suggesting that this is a general phenotype but is not specific to $\mathrm{H} 9 \mathrm{hESCs}$. Together, these data suggest that a certain amount of SMN-FL, but not SMN- $\Delta 7$, is crucially required for spinal motor neuron axonal outgrowth at an early stage during development.

Next, we performed the rescue experiment by excising the SMN-FL shRNA to determine whether the deficiency in the SMN gene expression directly contributes to the axonal outgrowth defects in SMN-FL-knockdown motor neurons. In the pLVTHM vector, there are LoxP sites flanking the shRNA that can be excised by introducing Cre. To excise the shRNA, we infected the caudalized neural progenitors (day 16) derived from SMN-FL RNAi hESCs with adenovirus containing Cre. After infection, the expression of SMN was examined in motor neuron progenitors (day 26) and motor neurons (day 33). As expected, the infection of Cre adenovirus successfully restored the expression of SMN-FL (Figure 4E) at the motor neuron progenitor and postmitotic motor neuron stages. Notably, the impaired axonal outgrowth of spinal motor neurons was also rescued when the SMN-FL levels were restored (Figure 4A and 4B). Together, these data confirm that the axonal outgrowth defects observed in cultures are directly caused by low expression of SMN-FL protein.

Considering that SMA is characterized by the specific degeneration of spinal motor neurons, we then tested whether the axonal outgrowth defects are specific to spinal motor neurons by generating forebrain neurons from control and SMN-FL-knockdown hESCs and comparing the axonal outgrowth. Our system [28] for specifying forebrain neurons from hESCs generated a high propor- tion of $\mathrm{FOXG}^{+}$telencephalic progenitors (Supplementary information, Figure S6), which differentiated into glutamatergic neurons, including $\mathrm{CTIP}^{+}$subcerebral projection neurons [30]. At the same time as tested in the motor neuron-enriched clusters ( 1 month after differentiation), forebrain progenitor-enriched clusters were plated on coverslips and the length of the $\mathrm{TAU}^{+}$axons was analyzed 2 days after plating. Notably, the axonal length was similar in the CTIP $2^{+}$forebrain neurons in the SMN-FL RNAi group to that in the control group (Figure $4 \mathrm{~F}$ and $4 \mathrm{G})$. Together, these data suggest the axonal outgrowth defects caused by the decreased level of SMN-FL are specific to spinal motor neurons.

\section{SMN-FL knockdown results in specific degeneration of} spinal motor neurons

Since knockdown of SMN-FL leads to impaired axonal outgrowth of spinal motor neurons specifically, it is possible that SMN-FL knockdown will result in specific motor neuron loss in the long-term culture. To test this, we cultured the motor neuron-enriched clusters on coverslips in the presence of neurotrophic factors [17] and compared the proportion of $\mathrm{HB}^{+}$motor neurons 6 weeks after differentiation from SMN-FL RNAi, SMN- $\Delta 7$ RNAi, and luciferase RNAi (control) hESCs. As shown in Figure 5A and 5B, the proportion of $\mathrm{HB}^{+}$motor neurons in the SMN-FL-knockdown group was about $10 \%$, which is much lower than that in the control group. In contrast, the proportion of spinal motor neurons derived from SMN- $\triangle 7$ RNAi hESCs remained at a comparable level to that from control hESCs (Figure 5A and 5B). These findings suggest that cultures derived from SMNFL RNAi hESCs exhibited loss of spinal motor neurons in long-term culture. To further confirm whether the neuronal loss is specific to spinal motor neurons, we cultured forebrain neurons for 6 weeks and compared the proportion of CTIP $2^{+}$neurons between control and SMNFL-knockdown groups. As shown in Figure 5C and 5D, knockdown of SMN-FL did not affect the proportion of $\mathrm{CTIP}^{+}$neurons 6 weeks after differentiation, suggesting that the neuronal degeneration caused by knocking down SMN-FL is specific to spinal motor neurons.

By reintroducing the SMN-FL gene to the cells with Cre, we showed that the axonal outgrowth defects can be rescued (Figure 4A and 4B). We further tested whether the subsequent motor neuron loss can be rescued in these cells. Cells were infected with Cre on day 16 and the proportion of $\mathrm{HB}^{+}$motor neurons was compared 6 weeks after differentiation. Interestingly, the proportion of $\mathrm{HB9}^{+}$motor neurons in Cre-infected cultures was significantly increased compared with that in the SMN-FLknockdown group and reached a comparable level to that 
A
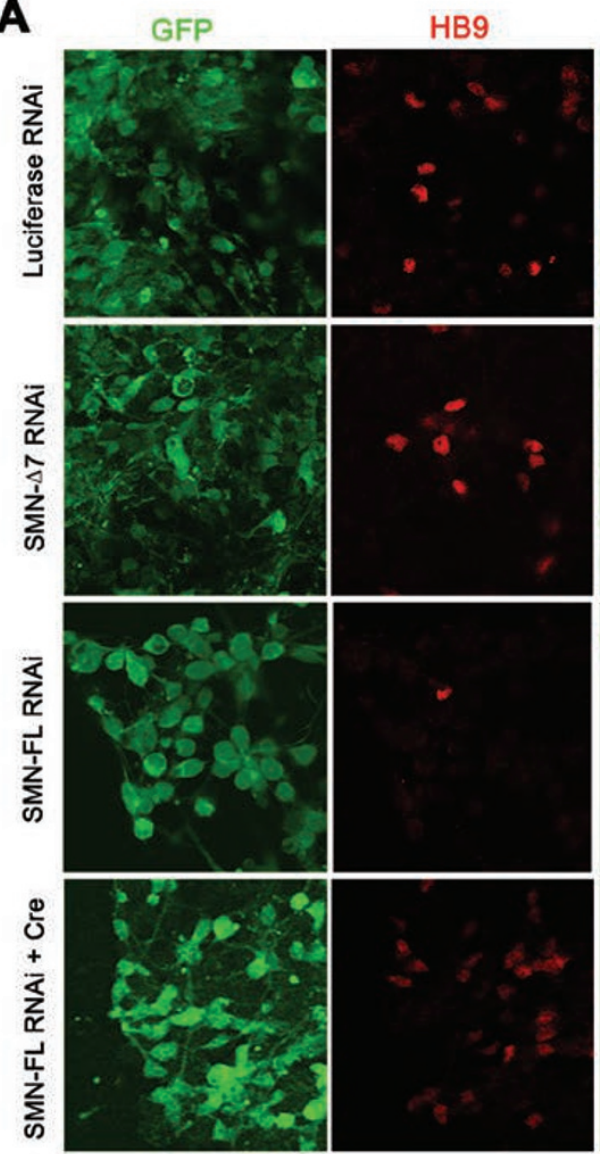
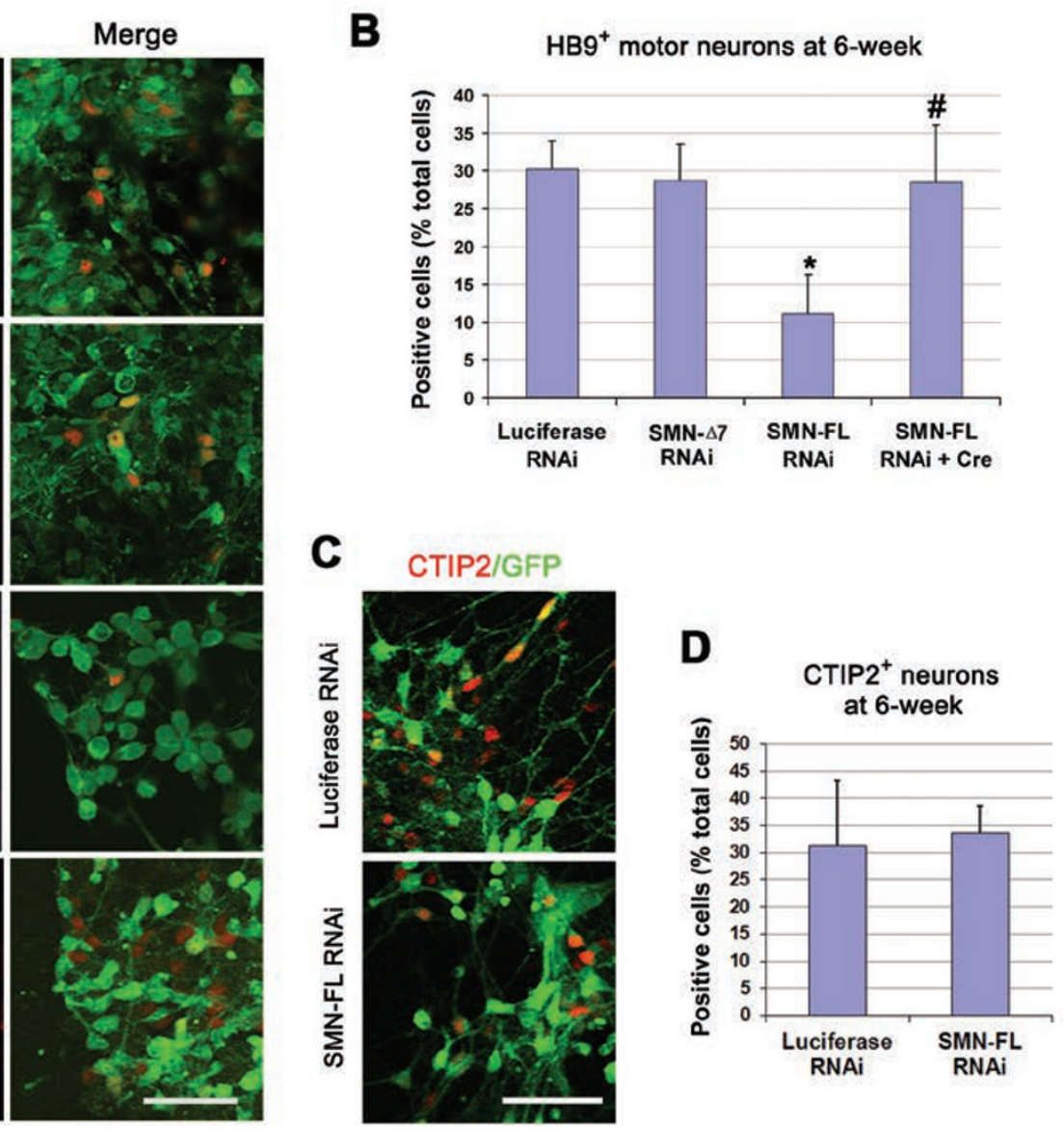

Figure 5 Specific loss of spinal motor neurons in long-term cultures differentiated from SMN-FL RNAi hESCs. (A) Six weeks after differentiation, the proportions of $\mathrm{HB9}^{+}$motor neurons were examined in cultures derived from control (luciferase RNAi) and SMN-knockdown (SMN- $\triangle 7$ RNAi, SMN-FL RNAi) hESCs. To rescue the expression of SMN-FL, we added Cre to the cultures on day 16 after differentiation (SMN-FL RNAi + Cre group). (B) Human motor neurons (HB9 ${ }^{+}$) derived from SMNFL RNAi hESCs showed a specific loss, which was rescued by the application of Cre. (C, D) Six weeks after differentiation, the proportion of CTIP2 ${ }^{+}$forebrain neurons was similar in cultures derived from control hESCs to that in cultures derived from SMN-FL-knockdown hESCs. Data are presented as mean \pm SD. ${ }^{*} P<0.05$ versus control (luciferase RNAi) group, ${ }^{\#} P<0.05$ versus SMN-FL RNAi group. Scale bar, $50 \mu \mathrm{m}$.

in control spinal motor neurons (Figure 5B). These data suggest that knockdown of SMN-FL leads to specific motor neuron degeneration, which can be rescued by excising the shRNA.

To exclude the off-target effect, we used another shRNA targeting SMN-FL and established stable hESC lines after viral infection and drug selection (Supplementary information, Figure S7A and S7B). These hESCs were differentiated into neural lineage and spinal motor neurons using the same protocol as described above. The expression of SMN protein in these SMN-knockdown ESCs and motor neuron cultures was significantly reduced compared with that in control cells (Supplementary information, Figure S7C). Similarly, these motor neuron- enriched cultures (day 33) showed impaired axonal outgrowth (Supplementary information, Figure S7D) and subsequent degeneration of $\mathrm{HB}^{+}$spinal motor neurons 6 weeks after differentiation (Supplementary information, Figure S7E).

To further exclude the off-target effect, we used another strategy to perform a rescue experiment by overexpressing SMN-FL through lentiviral infection. The SMN-FL in Plenti vector was made resistant to shRNA by changing the nucleotide sequence in the shRNA target region and its N-terminus was fused with FLAG tag (Figure 6A). Lentiviruses containing FLAG-GFP were used as a control. Similarly as in the Cre experiment, caudalized neural progenitors (day 16) were infected 
with lentiviruses containing FLAG-SMN. In contrast to control cells (infected with FLAG-GFP) that have very few axonal outgrowths, normal axonal outgrowth was observed in motor neuron cultures (day 33) that were infected with FLAG-SMN (Figure 6B). Six weeks after differentiation, the degeneration of $\mathrm{HB}^{+}$motor neurons was also rescued, as indicated by a significant increase in the proportion of $\mathrm{HB9}^{+}$cells after infection with the FLAG-SMN (Figure 6B and 6C). Taken together, these results suggest that the deficiency in the SMN-FL gene expression results in axonal outgrowth defects and neuronal degeneration in human spinal motor neurons specifically; of importance, these disease phenotypes can be efficiently rescued if SMN-FL is reintroduced into the cells.

Role of mitochondrial oxidative stress in motor neuron degeneration in SMN-FL-knockdown cultures

A recent study reported the dysfunction of mitochondria in NSC-43 cells whose SMN expression was knocked down using siRNA transfection [31], suggesting that mitochondrial defects may be implicated in this
SMA cell model. Therefore, to understand the mechanisms underlying the functional defects of spinal motor neurons caused by reduced level of SMN, we compared the mitochondrial superoxide production in cultures at motor neuron progenitor stage (26 days after differentiation) using MitoSOX staining. The MitoSOX ${ }^{+}$cells were significantly increased when SMN-FL, but not SMN- $\Delta 7$, was knocked down (Figure 7A and 7B). The application of Cre, which rescued the defects caused by SMN knockdown, decreased the generation of $\mathrm{MitoSOX}^{+}$cells in motor neuron cultures (Supplementary information, Figure S8). Interestingly, the proportion of $\mathrm{MitoSOX}^{+}$cells was similar in forebrain neuron cultures derived from control $(35 \% \pm 10 \%)$ or SMN-FL-knockdown hESCs $(33 \% \pm 11 \%)$. These data suggest that mitochondrial oxidative stress may be involved in the specific degeneration of spinal motor neurons in the culture.

Next, to confirm that oxidative stress specifically causes the motor neuron degeneration in our SMA model, we examined whether the application of $\mathrm{N}$-acetylcysteine (NAC), an antioxidant, has any beneficial effects on the motor neurons. NAC $(80 \mu \mathrm{g} / \mathrm{ml})$ was added to neural cul-
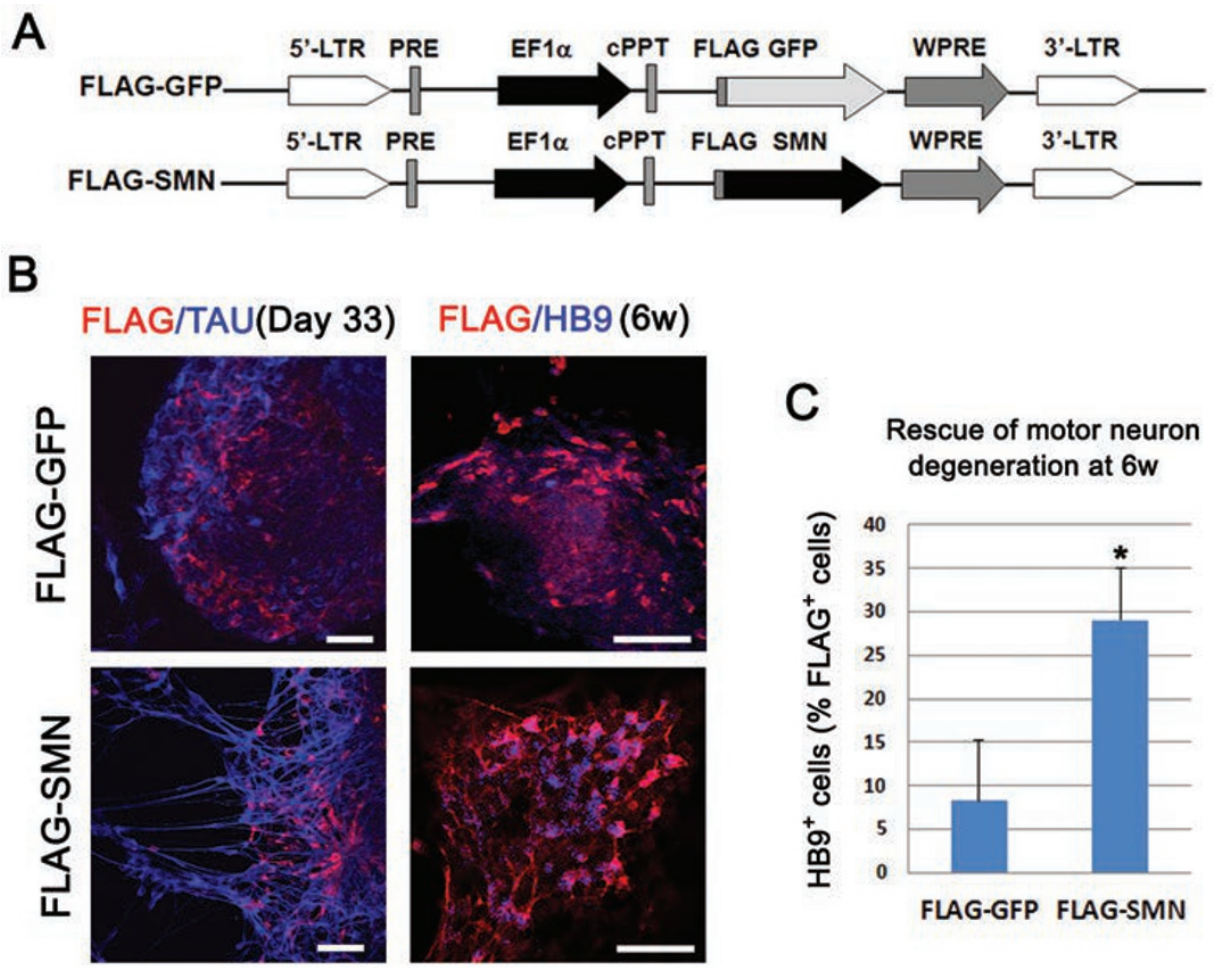

Figure 6 Overexpression of SMN rescued the phenotypes in SMN-FL-knockdown cultures. (A) Schematic map showing the Plenti vector containing FLAG-GFP or FLAG-SMN-FL. Rescue experiments were performed by infecting NE cells (day 16) with lentiviruses containing FLAG-SMN-FL. Lentiviruses containing FLAG-GFP were used as a control. (B) Motor neuronenriched cultures infected with FLAG-SMN showed normal axonal outgrowth on day 33. Infection of FLAG-SMN also rescued the motor neuron degeneration, as indicated by the increase in the proportion of $\mathrm{HB9}^{+}$motor neurons 6 weeks after differentiation (B, C). Data are presented as mean \pm SD. ${ }^{*} P<0.05$ versus FLAG-GFP groups by two-sided $t$-test. Scale bar, $50 \mu \mathrm{m}$. 
tures derived from SMN-FL RNAi hESCs from the NE stage to the motor neuron stage (day 17 to day 33). We then examined whether NAC can ameliorate the motor neuron degeneration detected in SMN-FL-knockdown cultures. Six weeks after differentiation, the proportion of $\mathrm{HB}^{+}$motor neurons in the NAC-treated group significantly increased (Figure 7C and 7D). Although NAC rescued the motor neuron degeneration caused by SMN knockdown, NAC had no effect on the impaired axonal outgrowth of motor neuron cultures (day 33) derived from SMN-FL RNAi hESCs (Figure 7E). These findings suggest that addition of NAC efficiently inhibits the motor neuron degeneration caused by the knockdown of SMN-FL in long-term culture and that different mechanisms may be implicated in axonal outgrowth defects and motor neuron degeneration in SMA.

To further investigate the mechanism underlying the protective role of NAC in the spinal motor neurons in our SMA model, taking into consideration that oxidative stress can lead to apoptosis, we compared the caspase 3/7 activity in motor neuron cultures. Luciferase RNAi and SMN-FL RNAi hESCs were differentiated into spinal motor neurons and NAC was added in the same time window (NE to $\mathrm{MN}$ stage), as shown earlier. In motor
A
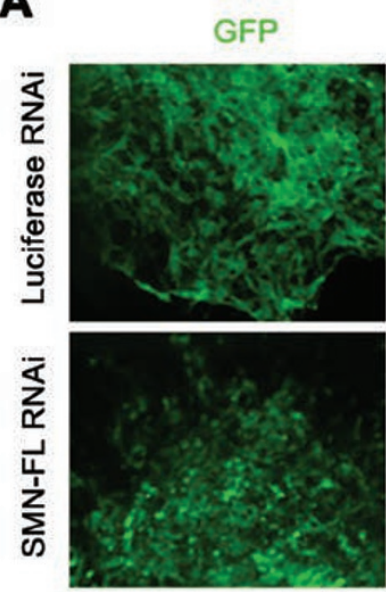

C
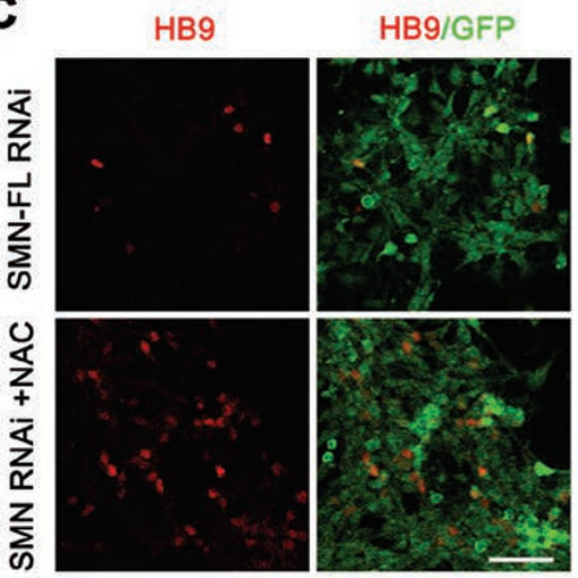

MitosoX
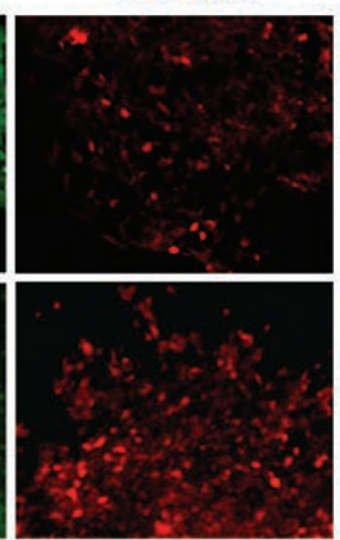

D

NAC rescued the loss of $\mathrm{HB}^{+}$ motor neurons at 6-week

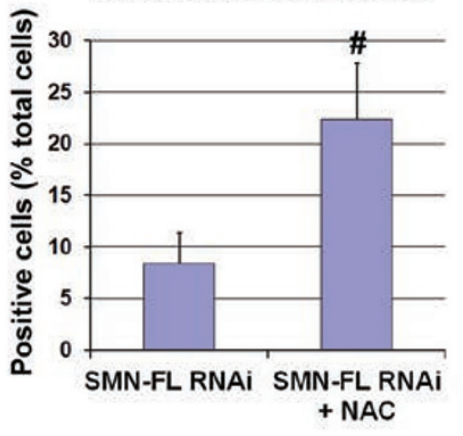

B

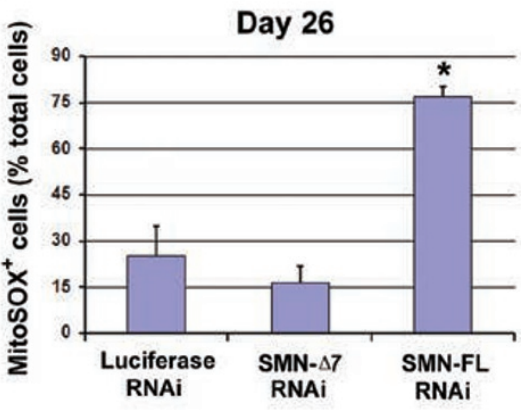

E

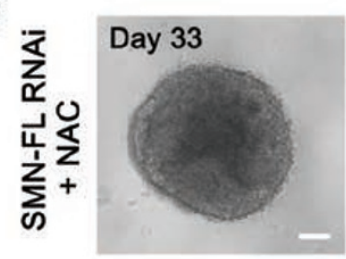

F

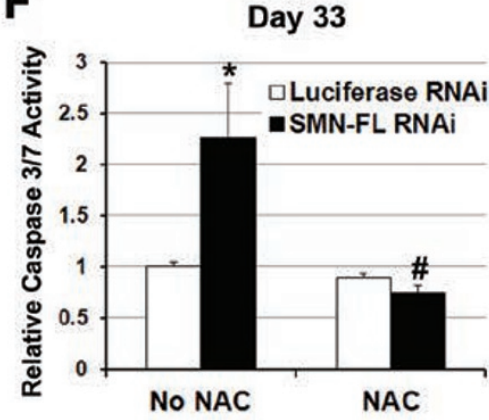

Figure 7 Effect of oxidative stress on motor neuron degeneration in SMN-FL-knockdown cultures. (A) Immunostaining showing the MitoSOX ${ }^{+}$cells in spinal motor neuron progenitor cultures in control and SMN-FL-knockdown groups. (B) The proportion of MitoSOX ${ }^{+}$cells was compared among groups. (C) NAC, a potent antioxidant, was applied to neural cultures derived from SMN-FL RNAi hESCs and the motor neuron population was examined by immunostaining 6 weeks after differentiation. (D) The proportion of $\mathrm{HB}^{+}$motor neurons was significantly increased after treatment with NAC. (E) Phase image showing the impaired axonal outgrowth in motor neuron cultures (day 33) in SMN-FL RNAi plus NAC treatment group. (F) The activity of caspase 3/7 was significantly increased in motor neuron progenitors derived from SMN-FL RNAi hESCs, and this increase was significantly inhibited by the application of NAC. Data are presented as mean \pm SD. ${ }^{*} P<0.05$ versus control (luciferase RNAi) group, ${ }^{\#} P<0.05$ versus SMN-FL RNAi group. Scale bar, $50 \mu \mathrm{m}$. 
neuron cultures (day 33), the caspase $3 / 7$ activity significantly increased in the SMN-FL-knockdown group as compared with that in the control group (Figure 7F), corresponding to neuronal loss in long-term cultures. Administration of NAC attenuated the caspase $3 / 7$ activity in SMN-FL-knockdown cultures to the basal level (Figure $7 \mathrm{~F}$ ). These data revealed that the addition of NAC completely rescued the SMN knockdown-induced apoptosis in spinal motor neuron cultures, suggesting that antioxidants may be a potential therapeutic strategy for SMA.

\section{Discussion}

SMA, one of the most common human autosomal recessive disorders, is caused by the decreased level of functional SMN protein, which leads to the specific degeneration of spinal motor neurons $[6,32]$. Humans have two $S M N$ genes that generate two forms of SMN protein, SMN-FL and SMN- 77 [3]. SMA patients have a decreased level of SMN-FL protein, but the level of SMN$\Delta 7$ protein remains similar to, or even higher than, that in normal individuals. To model SMA and tease out the role of these two different forms of SMN in motor neuron development, we established hESC lines with knockdown of SMN-FL or SMN- $\Delta 7$ transcripts. Knockdown of SMN-FL led to a decreased level of SMN-FL protein to about $20 \%$ of that in the control group, which is similar to what happened in type I SMA patients. As expected, knockdown of SMN-FL, but not SMN- $\Delta 7$, in human motor neurons resulted in a specific loss of spinal motor neurons in long-term culture, suggesting that SMN-FL is critical for maintaining the functions of spinal motor neurons derived from hESCs. Interestingly, during neural differentiation from normal hESCs, as we have shown, the expression of SMN-FL and SMN- $\Delta 7$ are specifically increased in spinal motor neurons, but not in forebrain neurons. Although it remains unclear whether the same changes occurred in vivo, it is possible that a high level of SMN is required to maintain the function of spinal motor neurons, contributing to the susceptibility of these neurons in SMA patients who experience improper SMN function.

The spinal motor neuron differentiation system resembles important stages during early development [17, 33], thus is very useful for studying the early changes and identifying phenotypes in our hESC-based SMA model. Using our well-established spinal motor neuron differentiation protocol [17, 27], we found that neural induction and initial motor neuron specification are not affected by SMN knockdown. Recent studies have revealed that SMN plays an important role in axonal outgrowth [14]; thus, we hypothesize that impaired axonal outgrowth may be an early pathological change in SMA patients. Indeed, the knockdown of SMN-FL specifically affects the axonal outgrowth of spinal motor neurons while the number of these neurons remains normal, after which the spinal motor neurons degenerate. These phenotypes were not observed in spinal motor neurons derived from SMN- 7 -knockdown hESCs, further confirming that SMN-FL is specifically required for proper motor neuron function. Notably, the axonal outgrowth and the neuronal degeneration of other types of neurons, such as forebrain projection neurons, are not affected by knocking down SMN-FL. These observations demonstrate that the phenotypes observed in our SMA model are specific to human spinal motor neurons, confirming the specificity of these disease-related phenotypes.

The mechanisms underlying the specific motor neuron loss in SMA patients are largely unclear. Studies using animal models and patient fibroblast cells have suggested that some pathways, such as mitochondrial function [31], are changed in SMA. However, whether these changes are a result or a cause for the disease is not clear. Using our SMA cell model, we revealed that at an earlier stage before the axonal outgrowth defects have been observed, the level of mitochondrial oxidative stress was significantly and specifically increased in motor neuron cultures, suggesting that this process is more likely causative in SMA. This brings up a potential target for the treatment of SMA with antioxidants. By applying NAC, an antioxidant in SMA-mimicking neural cultures, we found that spinal motor neuron apoptosis and subsequent degeneration, but not axonal outgrowth defects, were greatly ameliorated. These results suggest that oxidative stress may not be a causative factor for early axonal outgrowth defects. However, as motor neuron degeneration is the endpoint of SMA pathology, treatment with antioxidants may greatly ameliorate motor neuron degeneration, although the cells may still display some axonal phenotypes. It would be intriguing to test whether antioxidants could exert a protective effect solely at a certain time window in animal models or whether it would need to be applied together with anti-axonopathy agents.

Considering that SMA is a complicated neurodegenerative disease, it is noteworthy that other mechanisms may also participate in the spinal motor neuron degeneration that characterizes this disorder. For example, neurotrophic factors are critical for the survival of spinal motor neurons [34-36]. It is possible that SMN disrupts the responses of motor neurons to trophic factors, which needs to be further investigated. In our culture, astrocytes were not generated at an early differentiation stage when the motor neurons show axonal outgrowth defects. However, astrocytes were present at a later stage compared to 
neurons (Supplementary information, Figure S6), which coincides with both previous studies $[37,38]$ and the timing that occurs during in vivo development [39]. This also raises another interesting question about whether the phenotype of SMA in the culture dish is cell autonomous. It is important to perform further experiments to study whether other cell types, such as astrocytes, and deficiencies in forming functional synaptic connections, also contribute to motor neuron degeneration.

With the development of culture systems to efficiently generate neural lineage and functional neurons from human pluripotent stem cells, these systems have been used to model neurogenetic disorders through the manipulation of disease-determining genes in hESCs or the generation of patient-specific iPSCs [26, 40-44]. To be an ideal disease model, the culture system should be able to generate the target neurons efficiently and to recapitulate the in vivo pathological process in these target neurons. More importantly, it is critical to evaluate whether the pathological and functional changes are cell type specific and are directly caused by the gene defects. In this study, to mimic the pathological changes in SMA, we knocked down SMN-FL in hESCs and differentiated these ESCs into spinal motor neurons. These SMA-like spinal motor neurons exhibited impaired axonal outgrowth and subsequent neuronal degeneration. An important aspect of these disease phenotypes is that they are specific to spinal motor neurons and can be rescued by restoring the $S M N$ gene. Thus, we have established a closely representative cell model for SMA. This model, as indicated by our study, offers a unique system for exploring the mechanisms underlying motor neuron degeneration in SMA and for developing a potential treatment for this devastating disease.

\section{Materials and Methods}

\section{Culture of hESCs}

Human ESCs (H9 and H1, passages 15 to 45) were cultured on a feeder layer of irradiated mouse embryonic fibroblasts (MEFs) in hESC medium consisting of Dulbecco's modified Eagle's medium (DMEM)/F12, knockout serum replacement, 2-mercaptoethanol, L-glutamine, and non-essential amino acids as previously described [15]. The hESC cultures were passaged weekly and routinely screened with VenorGeM (Sigma-Aldrich) to ensure that they were free of mycoplasma contamination.

\section{Lentivirus production and transduction of hESCs}

Knockdown of SMN was achieved using lentiviruses (pLVTHM backbone, Addgene) that contain shRNAs targeting human SMNFL and SMN- $\Delta 7$ (Supplementary information, Figure S2), a strategy similar to the one that we described for knocking down other genes [28, 45]. To produce lentivirus, pLVTHM, psPAX2, and pMD2.G (VSV-G envelope protein) were cotransfected to
HEK293FT cells (Invitrogen) using the calcium phosphate method. For transduction of ESCs, hESCs were normally passaged and pelleted by brief centrifugation. Cell pellets were then incubated with $100 \mu \mathrm{l}$ of concentrated virus $\left(\sim 10^{6}\right.$ transducing units $\left./ \mathrm{ml}\right)$ at $37^{\circ} \mathrm{C}$ for $30 \mathrm{~min}$. The virus and cell mixture was then plated to a MEF feeder layer overnight before changing medium on the next day. The SMN RNAi cells were selected by expression of GFP and resistance to BSD. The RNAi cells were selected and replated for several passages to yield a purer population.

To overexpress shRNA-resistant SMN-FL, we constructed Plenti vectors containing mutant SMN-FL or GFP (as a control) (Figure 6A). A FLAG tag was fused to the N-terminus of GFP or SMN-FL (Figure 6A). To introduce the FLAG and mutations in SMN-FL, we used SMN-FL cDNA in pGEM-T Easy plasmid as a template for PCR amplification with the following primers: 5'-GATCGGATCCACCGGTCGCCACCATGGACTACAAAGACGATGACGATAAGATGGCGATGAGCAGCGGCGGC-3' (forward), 5'-AGTCGCGGCCGCTTAATTTAA $\underline{A}$ GAGTGC-

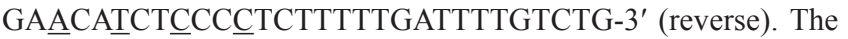
FLAG and changed nucleotides are underlined. These changes did not alter the amino acid sequence and were confirmed by DNA sequencing. Lentiviruses were generated using the same method and were then utilized for rescue experiments.

\section{Neural differentiation from $h E S C s$}

The procedure for generating neuroepithelial cells and spinal motor neurons from hESCs was essentially the same as described $[17,38]$. Briefly, hESCs were induced to neural lineage by forming ESC aggregations and then culturing in neural medium. The early NE cells were formed around 8-10 days after differentiation from hESCs, which exhibited columnar morphology and started to organize into a rosette-like structure. Human ESC-derived NE cells at day 10 were then treated with RA $(0.1 \mu \mathrm{M})$ for caudalization in a neural medium, which consisted of DMEM/F-12 medium, N2 supplement, and heparin. One week later (day 17), the posteriorized NE cells were isolated. For specifying spinal motor neurons, these NE clusters were suspended in the same neural medium supplemented with B27, RA and purmorphamine. To generate forebrain neurons, the neuroepithelial cells were cultured in basic neural medium without any caudalizing factors (RA, basic fibroblast growth factor) [28]. These rostral NE cells were also isolated at day 17 and suspended in the same neural medium with B27. For terminal differentiation, the neural progenitor-enriched clusters were plated onto ornithine/laminin-coated coverslips in Neurobasal medium (Invitrogen) supplemented with N2 and B27.

\section{Immunocytochemistry and quantification}

Coverslips were fixed with $4 \%$ paraformaldehyde and immunohistochemistry was performed as previously described [17]. Antigen-antibody reactions were developed by appropriate fluorescence-conjugated secondary antibodies. Nuclei were visualized by Hoechst staining. Primary antibodies used in this study included goat anti-Olig2 (1:400, Santa Cruz Biotechnology), mouse antiHB9 (1:50, Developmental Studies Hybridoma Bank, DSHB), rabbit anti-Tau (1:100, Sigma), rat anti-Ctip2 (1:2 000, Abcam), rabbit anti- $\beta$ III-tubulin (1:5 000, Covance), mouse anti-Pax6 (1:5 000, DSHB), goat anti-ChAT (1:100, Chemicon), mouse anti-Bassoon (1:200, Stressgen), guinea pig anti-vGLUT1(1:2 000, Millipore), rabbit anti-MAP2 (1:2 000, Millipore), mouse anti-S100 $\beta$ (1:1 
000, Abcam), mouse anti-FLAG M2 (1:1 000, Sigma), and rabbit anti-FLAG (1:200, Cell Signaling). Images were collected using a Zeiss camera mounted onto Zeiss Axiovert 200M fluorescence microscope or Zeiss LSM 510 Meta confocal microscope.

The population of OLIG2, HB9 and CTIP2-expressing cells among total differentiated cells (GFP labeled) was counted as described previously [28]. Briefly, the Zeiss microscope was used to capture images. At least 4 fields of each coverslip were chosen and counted using a Metamorph software program (Universal Imaging Corporation, Downingtown, PA, USA) by an observer blinded to the experimental conditions. For each group, 3-6 coverslips were counted. Data were expressed as Mean \pm SD.

For analyzing the axonal outgrowth of $\mathrm{HB}^{+}$spinal motor neurons, the lengths of $\mathrm{TAU}^{+}$axons were measured using the MetaMorph Microscopy Automation \& Image Analysis Software (Molecular Devices) by an observer blinded to the experimental conditions. At least 30 cells were measured in each group. The average lengths of axons were calculated and compared between different groups using Tukey's studentized range test (see statistical analysis).

\section{$R N A$ isolation, $R T-P C R$ and $q P C R$}

Total RNA was extracted from cultures at different stages using TRIzol, treated with DNase to remove genomic DNA according to the supplier's protocol, and used as templates for the PCR reaction. Quantitative PCR (qPCR) reactions were performed in a $20-\mu 1$ mixture containing cDNA, primers, and $1 \times$ SYBR GREEN PCR Master mix (Bio-Rad). Standard curves and melting curves were plotted for each set of primers to confirm that only 1 amplicon was generated at the same efficiency as $G A P D H$, a housekeeping gene. Expression levels of the mRNA were calculated using the comparative CT method. The following primers were used: SMN-FL, 5'ATGTTAATTTCATGGTACATG-3', 5' - GGAATGTGAGCACCTTCCTTC-3'; SMN- $\Delta$ 7, 5'-ATATGGAAATGCTGGCATAGAG-3', 5'-TCCAGATCTGTCTGATCGTTTC-3'; GAPDH, 5'-ATGACATCAAGAAGGTGGTG-3', 5'-CATACCAGGAAATGAGCTTG-3'.

\section{Western blot}

Cell pellets were collected and resuspended in lysis buffer with protease inhibitor cocktail (Sigma), and then passed through a 28.5-gauge needle and lysed overnight. The particulate fraction was removed by centrifugation. Proteins $(10-20 \mu \mathrm{g})$ were separated on $10 \%$ SDS-PAGE and subjected to immunoblotting analysis. Both blocking and antibody incubations were carried out in Tris-

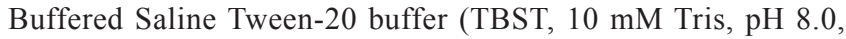
$150 \mathrm{mM} \mathrm{NaCl}, 0.05 \%$ Tween 20, pH 8.0) containing 5\% nonfat dry milk. Primary antibodies used were mouse anti-actin (1:1 000, Sigma) and mouse anti-SMN1 (1:1 000, Abnova). Horseradish peroxidase-conjugated secondary antibodies were detected with Western Lighting Chemiluminescence Reagent Plus (Pierce).

\section{FACS analysis}

To prepare samples for FACS, NE cells derived from hESCs were dissociated into single cells using Accutase as described previously [24]. Cells were fixed and permeablized with ice-cold $0.1 \%$ paraformaldehyde for $10 \mathrm{~min}$ and $90 \%$ methanol for $30 \mathrm{~min}$. After incubating with anti-PAX6 antibody ( $1 \mu \mathrm{g}$ per $10^{6}$ cells $)$ or a normal mouse IgG as a negative control overnight, NE cells were then washed and incubated with Alexa Fluor 594-conjugated donkey anti-mouse IgG for $1 \mathrm{~h}$ followed by three washing steps. The samples were analyzed on a Becton Dickinson FACSCalibur instrument, and the ratio of $\mathrm{PAX}^{+}$cells was calculated by using CellQuest Pro software (BD Biosciences).

\section{Adenovirus production and infection}

High-titer adenovirus was generated using a protocol similar to that described by the manufacturer. Briefly, adenovirus containing CMV-Cre-IRES-GFP (Vector Biolabs) was used to infect HEK293A (Invitrogen) cells at a multiplicity of infection (MOI) of $5-10$. When the cytopathic effects were observed and about $80 \%$ of the cells had detached (about 3 days after infection), cells were collected and pelleted. The cell pellets were then resuspended in phosphate buffered saline and subjected to three freeze-thaw cycles. After the third cycle, cells were centrifuged and the supernatant (crude lysate) was collected. The titer of this crude lysate was tested using HEK293A cells. To infect neural cultures, we added adenovirus to the culture medium ( $1: 400$ dilution; MOI $\sim 50)$ for $12 \mathrm{~h}$, followed by a change of fresh medium.

Analysis of mitochondrial superoxide and caspase 3/7 activity Mitochondrial superoxide was detected by using MitoSOX Red according to the manufacturer's protocol (Molecular Probes, Invitrogen). MitoSOX Red was prepared as a $5 \mathrm{mM}$ stock solution in DMSO immediately before the experiments. For detecting the superoxide levels, cells cultured on coverslips in a 24 -well plate were incubated with this solution at $5 \mu \mathrm{M}$ for $10 \mathrm{~min}$ and then washed with Hank's balanced salt solution. The oxidation by superoxide produces red fluorescence in live cells, and images were taken with an inverted Zeiss microscope. The MitoSOX ${ }^{+}$cells (red) were then counted by using a Metamorph software program as described above.

For measurements of the activities of caspases 3 and 7, the Caspase-Glo 3/7 Assay (Promega) was carried out according to the manufacturer's instructions. Briefly, motor neuron progenitorenriched cultures were dissociated with Accutase (Invitrogen) and seeded into 96-well plates at 5000 cells/well in $50 \mu$ of caspase-3/7 reagent. After incubation for $1 \mathrm{~h}$ at room temperature, luminescence from each well was then measured using Wallac Victor2 1420 MultiLabel Counter.

\section{Statistical analysis}

The statistical significance in mean values between multiple sample groups was examined with two-sided Tukey's studentized range test after one-way ANOVA. Two-sided $t$-test was used to examine the statistical significance between two sample groups. The significance level was defined as $P<0.05$, and all significance tests were conducted using SAS 9.1 (SAS Institute).

\section{Acknowledgments}

We are grateful to Xiaonan Xin, Mary L Stover, and Alex Lichtler for their technical support on making adenovirus. We also thank Yoshiki Sasai (Kyoto University, Japan) for generously providing the FOXG1 antibody. This study was supported by Connecticut Stem Cell Research Grants (08-SCB-UCHC-022 and $11 \mathrm{SCB} 24$ to XJ L), and Science and Technology Commission of Shanghai Municipality of China (11PJ1410000 to XZ). 


\section{References}

1 Pearn JH, Gardner-Medwin D, Wilson J. A clinical study of chronic childhood spinal muscular atrophy. A review of 141 cases. J Neurol Sci 1978; 38:23-37.

2 Lefebvre S, Burglen L, Reboullet S, et al. Identification and characterization of a spinal muscular atrophy-determining gene. Cell 1995; 80:155-165.

3 Rochette CF, Gilbert N, Simard LR. SMN gene duplication and the emergence of the SMN2 gene occurred in distinct hominids: SMN2 is unique to Homo sapiens. Hum Genet 2001; 108:255-266.

4 Lorson CL, Hahnen E, Androphy EJ, Wirth B. A single nucleotide in the $S M N$ gene regulates splicing and is responsible for spinal muscular atrophy. Proc Natl Acad Sci USA 1999; 96:6307-6311.

5 Monani UR, Lorson CL, Parsons DW, et al. A single nucleotide difference that alters splicing patterns distinguishes the SMA gene SMN1 from the copy gene SMN2. Hum Mol Genet 1999; 8:1177-1183.

6 Wirth B, Brichta L, Hahnen E. Spinal muscular atrophy: from gene to therapy. Semin Pediatr Neurol 2006; 13:121-131.

7 Liu Q, Dreyfuss G. A novel nuclear structure containing the survival of motor neurons protein. EMBO J 1996; 15:35553565.

8 Young PJ, Le TT, thi Man N, Burghes AH, Morris GE. The relationship between SMN, the spinal muscular atrophy protein, and nuclear coiled bodies in differentiated tissues and cultured cells. Exp Cell Res 2000; 256:365-374.

9 Fischer U, Liu Q, Dreyfuss G. The SMN-SIP1 complex has an essential role in spliceosomal snRNP biogenesis. Cell 1997; 90:1023-1029.

10 Pellizzoni L, Kataoka N, Charroux B, Dreyfuss G. A novel function for SMN, the spinal muscular atrophy disease gene product, in pre-mRNA splicing. Cell 1998; 95:615-624.

11 Winkler C, Eggert C, Gradl D, et al. Reduced U snRNP assembly causes motor axon degeneration in an animal model for spinal muscular atrophy. Genes Dev 2005; 19:2320-2330.

12 Fan L, Simard LR. Survival motor neuron (SMN) protein: role in neurite outgrowth and neuromuscular maturation during neuronal differentiation and development. Hum Mol Genet 2002; 11:1605-1614.

13 Zhang HL, Pan F, Hong D, et al. Active transport of the survival motor neuron protein and the role of exon-7 in cytoplasmic localization. $J$ Neurosci 2003; 23:6627-6637.

14 Rossoll W, Jablonka S, Andreassi C, et al. Smn, the spinal muscular atrophy-determining gene product, modulates axon growth and localization of beta-actin mRNA in growth cones of motoneurons. J Cell Biol 2003; 163:801-812.

15 Thomson JA, Itskovitz-Eldor J, Shapiro SS, et al. Embryonic stem cell lines derived from human blastocysts. Science 1998; 282:1145-1147.

16 Lee H, Shamy GA, Elkabetz Y, et al. Directed differentiation and transplantation of human embryonic stem cell-derived motoneurons. Stem Cells 2007; 25:1931-1939.

17 Li XJ, Du ZW, Zarnowska ED, et al. Specification of motoneurons from human embryonic stem cells. Nat Biotechnol 2005; 23:215-221.

18 Singh Roy N, Nakano T, Xuing L, et al. Enhancer-specified
GFP-based FACS purification of human spinal motor neurons from embryonic stem cells. Exp Neurol 2005; 196:224-234.

19 Perrier AL, Tabar V, Barberi T, et al. Derivation of midbrain dopamine neurons from human embryonic stem cells. Proc Natl Acad Sci USA 2004; 101:12543-12548.

20 Roy NS, Cleren C, Singh SK, et al. Functional engraftment of human ES cell-derived dopaminergic neurons enriched by coculture with telomerase-immortalized midbrain astrocytes. Nat Med 2006; 12:1259-1268.

21 Yan Y, Yang D, Zarnowska ED, et al. Directed differentiation of dopaminergic neuronal subtypes from human embryonic stem cells. Stem Cells 2005; 23:781-790.

22 Boulting GL, Kiskinis E, Croft GF, et al. A functionally characterized test set of human induced pluripotent stem cells. Nat Biotechnol 2011; 29:279-286.

$23 \mathrm{Hu}$ BY, Weick JP, Yu J, et al. Neural differentiation of human induced pluripotent stem cells follows developmental principles but with variable potency. Proc Natl Acad Sci USA 2010; 107:4335-4340.

24 Zeng H, Guo M, Martins-Taylor K, et al. Specification of region-specific neurons including forebrain glutamatergic neurons from human induced pluripotent stem cells. PLoS One 2010; 5:e11853.

25 Chang T, Zheng W, Tsark W, et al. Brief report: phenotypic rescue of induced pluripotent stem cell-derived motoneurons of a spinal muscular atrophy patient. Stem Cells 2011; 29:2090-2093.

26 Ebert AD, Yu J, Rose FF Jr, et al. Induced pluripotent stem cells from a spinal muscular atrophy patient. Nature 2009; 457:277-280.

27 Li XJ, Hu BY, Jones SA, et al. Directed differentiation of ventral spinal progenitors and motor neurons from human embryonic stem cells by small molecules. Stem Cells 2008; 26:886-893.

28 Li XJ, Zhang X, Johnson MA, et al. Coordination of sonic hedgehog and Wnt signaling determines ventral and dorsal telencephalic neuron types from human embryonic stem cells. Development 2009; 136:4055-4063.

29 Pankratz MT, Li XJ, Lavaute TM, et al. Directed neural differentiation of human embryonic stem cells via an obligated primitive anterior stage. Stem Cells 2007; 25:1511-1520.

30 Arlotta P, Molyneaux BJ, Chen J, et al. Neuronal subtypespecific genes that control corticospinal motor neuron development in vivo. Neuron 2005; 45:207-221.

31 Acsadi G, Lee I, Li X, et al. Mitochondrial dysfunction in a neural cell model of spinal muscular atrophy. $J$ Neurosci Res 2009; 87:2748-2756.

32 Monani UR. Spinal muscular atrophy: a deficiency in a ubiquitous protein; a motor neuron-specific disease. Neuron 2005; 48:885-896.

33 Li XJ, Zhang SC. In vitro differentiation of neural precursors from human embryonic stem cells. Methods Mol Biol 2006; 331:169-177.

34 Brunet N, Tarabal O, Portero-Otin M, et al. Survival and death of mature avian motoneurons in organotypic slice culture: trophic requirements for survival and different types of degeneration. J Comp Neurol 2007; 501:669-690.

35 Henderson CE, Phillips HS, Pollock RA, et al. GDNF: a potent survival factor for motoneurons present in peripheral 
nerve and muscle. Science 1994; 266:1062-1064.

36 Sendtner M, Pei G, Beck M, Schweizer U, Wiese S. Developmental motoneuron cell death and neurotrophic factors. Cell Tissue Res 2000; 301:71-84.

37 Johnson MA, Weick JP, Pearce RA, Zhang SC. Functional neural development from human embryonic stem cells: accelerated synaptic activity via astrocyte coculture. $J$ Neurosci 2007; 27:3069-3077.

38 Zhang SC, Wernig M, Duncan ID, Brustle O, Thomson JA. In vitro differentiation of transplantable neural precursors from human embryonic stem cells. Nat Biotechnol 2001; 19:11291133.

39 Qian X, Shen Q, Goderie SK, et al. Timing of CNS cell generation: a programmed sequence of neuron and glial cell production from isolated murine cortical stem cells. Neuron 2000; 28:69-80.

40 Chamberlain SJ, Chen PF, Ng KY, et al. Induced pluripotent stem cell models of the genomic imprinting disorders Angelman and Prader-Willi syndromes. Proc Natl Acad Sci USA 2010; 107:17668-17673.

41 Karumbayaram S, Kelly TK, Paucar AA, et al. Human embryonic stem cell-derived motor neurons expressing SOD1 mutants exhibit typical signs of motor neuron degeneration linked to ALS. Dis Model Mech 2009; 2:189-195.

42 Kiskinis E, Eggan K. Progress toward the clinical application of patient-specific pluripotent stem cells. J Clin Invest 2010; 120:51-59.

43 Lee G, Papapetrou EP, Kim H, et al. Modelling pathogenesis and treatment of familial dysautonomia using patient-specific iPSCs. Nature 2009; 461:402-406.

44 Walsh RM, Hochedlinger K. Modeling Rett syndrome with stem cells. Cell 2010; 143:499-500.

45 Zhang X, Huang CT, Chen J, et al. Pax6 is a human neuroectoderm cell fate determinant. Cell Stem Cell 2010; 7:90-100.

(Supplementary information is linked to the online version of the paper on the Cell Research website.) 